\title{
CAUCHY PROBLEM IN GENERALIZED GEVREY CLASSES
}

\author{
DANIELA CALVO \\ Dipartimento di Matematica "L. Tonelli" \\ Università di Pisa \\ Via Buonarroti, 2, 56127 Pisa, Italy \\ E-mail: calvo@mail.dm.unipi.it
}

\begin{abstract}
In this work we present a class of partial differential operators with constant coefficients, called multi-quasi-hyperbolic and defined in terms of a complete polyhedron. For them we obtain the well-posedness of the Cauchy problem in generalized Gevrey classes determined by means of the same polyhedron. We present some necessary and sufficient conditions on the operator in order to be multi-quasi-hyperbolic and give some examples.
\end{abstract}

Introduction. We want to generalize a theorem of well-posedness of the Cauchy problem in Gevrey classes $G^{s}, 1<s<\infty$ (for definition and properties of $G^{s}$ see for example Rodino [14]). In fact, solvability of the Cauchy problem in Gevrey spaces has been obtained for weakly hyperbolic operators with constant coefficients, namely for the class of the so-called $s$-hyperbolic operators (see Cattabriga [4], Hörmander [10], Rodino [14]).

This result can be extended to operators with variable coefficients, for example we refer to the important contributions of Bronstein [2], Steinberg [15] and Kajitani [11].

Here, remaining in the frame of constant coefficients, we want to improve the previous result in order to assure the solvability of the Cauchy problem in generalized Gevrey classes $G^{s \mathcal{P}}$. These classes are defined in terms of a complete polyhedron $\mathcal{P}$. We will treat them following Zanghirati [17], Corli [7] and give a characterization also by means of Fourier transform, see Section 1.

Let us observe that $G^{s} \subset G^{s \mathcal{P}}$, cf. Remark 3. This allows in fact a more general result of well-posedness, by enlarging the class of the admissible data, but we need to ask some conditions on the operator, in particular to this end we define multi-quasi-hyperbolic operators as in the following:

2000 Mathematics Subject Classification: Primary 35L40.

The paper is in final form and no version of it will be published elsewhere. 
Definition 1. Let $1<s<\infty$ and let $\mathcal{P}$ be a complete polyhedron. We say that a differential operator with constant coefficients in $\mathbf{R}_{x}^{n} \times \mathbf{R}_{t}$ of the form

$$
P\left(D_{t}, D_{x}\right)=D_{t}^{m}+\sum_{|\nu|+j \leq m} a_{\nu j} D_{x}^{\nu} D_{t}^{j}
$$

is multi-quasi-hyperbolic of order $s$ with respect to $\mathcal{P}$ if there exists a constant $C>0$ such that for $(\lambda, \xi) \in \mathbf{C} \times \mathbf{R}^{n}$ the condition

$$
P(\lambda, \xi)=\lambda^{m}+\sum_{|\nu|+j \leq m} a_{\nu j} \xi^{\nu} \lambda^{j}=0
$$

implies

$$
\Im \lambda \geq-C|\xi|_{\mathcal{P}}^{1 / s}
$$

where $|\xi|_{\mathcal{P}}$ is the weight associated to $\mathcal{P}$, see Section 1 for the definition.

We will see some properties of multi-quasi-hyperbolic operators and give some necessary and sufficient conditions and examples in Section 2. Finally, for them we shall present a theorem of well-posedness in generalized Gevrey classes in Section 3, giving a regularity result of the solution in the space and time variables; for the proof we address to Calvo [3]. With respect to [3], here we prove stronger results concerning the characterization of multi-quasi-hyperbolic operators and give some examples, see Section 2 for details.

1. Complete polyhedra and generalized Gevrey classes. Here we recall the definition of complete polyhedra and generalized Gevrey classes following Zanghirati [17], Corli [7] and give an equivalent characterization based on the Fourier transform, as in Calvo [3].

A convex polyhedron $\mathcal{P}$ in $\mathbf{R}^{n}$ is the convex hull of a finite set of points in $\mathbf{R}^{n}$. There is uniquely determined by $\mathcal{P}$ a finite $\operatorname{set} \mathcal{V}(\mathcal{P})$ of linearly independent points, called the set of vertices of $\mathcal{P}$, as the smallest set whose convex hull is $\mathcal{P}$.

Moreover, if $\mathcal{P}$ has a non-empty interior, there exists a finite set

$$
\mathcal{N}(\mathcal{P})=\mathcal{N}_{0}(\mathcal{P}) \cup \mathcal{N}_{1}(\mathcal{P})
$$

such that

$$
\begin{aligned}
& |\nu|=1 \text { for every } \nu \in \mathcal{N}_{1}(\mathcal{P}) \\
& \qquad \begin{array}{l}
\text { and } \quad \mathcal{P}=\left\{z \in \mathbf{R}^{n}: \forall \nu \in \mathcal{N}_{0}(\mathcal{P}) \nu \cdot z \geq 0 \wedge \forall \nu \in \mathcal{N}_{1}(\mathcal{P}) \nu \cdot z \leq 1\right\}
\end{array}
\end{aligned}
$$

The boundary of $\mathcal{P}$ is made of faces $\mathcal{F}_{\nu}$ of the equation

$$
\begin{aligned}
& \nu \cdot z=0 \text { if } \nu \in \mathcal{N}_{0}(\mathcal{P}) \\
& \nu \cdot z=1 \text { if } \nu \in \mathcal{N}_{1}(\mathcal{P}) .
\end{aligned}
$$

Definition 2. A complete polyhedron is a convex polyhedron $\mathcal{P} \subset \mathbf{R}_{+}^{n}$ satisfying the following conditions:

1. $\mathcal{V}(\mathcal{P}) \subset \mathbf{N}^{n}$ (i.e. all vertices have integer coordinates);

2. the origin $(0,0, \ldots, 0)$ belongs to $\mathcal{P}$;

3. $\operatorname{dim}(\mathcal{P})=n$; 
4. $\mathcal{N}_{0}(\mathcal{P})=\left\{e_{1}, e_{2}, \ldots, e_{n}\right\}$, with $e_{j}=(\underbrace{0,0, \ldots, 0}_{j-1}, 1, \underbrace{0, \ldots, 0}_{n-j}) \in \mathbf{R}^{n}$ for $j=1, \ldots, n$; 5. $\mathcal{N}_{1}(\mathcal{P}) \subset \mathbf{R}_{+}^{n}$.

We note that condition 5 means that the set

$$
Q(x)=\left\{y \in \mathbf{R}^{n}: 0 \leq y \leq x\right\} \subset \mathcal{P} \text { if } x \in \mathcal{P}
$$

and that if $s$ belongs to a face of $\mathcal{P}$ and $r>s$ (i.e. each component $r_{j}>s_{j}, j=1, \ldots, n$ ), then $r \notin \mathcal{P}$.

We can consider also polyhedra with rational vertices instead of integer vertices and obtain the same theory. We now give some notation related to a convex polyhedron $\mathcal{P}$.

We define: $\mathcal{L}(\mathcal{P})$ - the cardinality of $\mathcal{N}_{1}(\mathcal{P})$

$\forall \nu \in \mathcal{N}_{1}(\mathcal{P}) \mathcal{F}_{\nu}(\mathcal{P})=\{s \in \mathcal{P}: \nu \cdot s=1\}$ - a face of $\mathcal{P} ;$ $\mathcal{F}=\bigcup_{\nu \in \mathcal{N}_{1}(\mathcal{P})} \mathcal{F}_{\nu}(\mathcal{P})$ - the boundary of $\mathcal{P}$

$\mathcal{V}(\mathcal{P})$ - the set of vertices of $\mathcal{P}$;

$\delta \mathcal{P}=\left\{s \in \mathbf{R}_{+}^{n}: \delta^{-1} s \in \mathcal{P}\right\}, \delta>0$

$k(s, \mathcal{P})=\inf \left\{t>0: t^{-1} s \in \mathcal{P}\right\}=\max _{\nu \in \mathcal{N}_{1}(\mathcal{P})} \nu \cdot s, s \in \mathbf{R}_{+}^{n}$.

Now let $\mathcal{P}$ be a complete polyhedron. We say that

$\mu_{j}(\mathcal{P})=\max _{\nu \in \mathcal{N}_{1}(\mathcal{P})} \nu_{j}^{-1}$

$\mu=\mu(\mathcal{P})=\max _{j=1, \ldots, n} \mu_{j}$ is the formal order of $\mathcal{P}$;

$\mu^{(0)}(\mathcal{P})=\min _{\gamma \in \mathcal{V}(\mathcal{P}) \backslash\{0\}}|\gamma| \quad$ is the minimum order of $\mathcal{P}$;

$\mu^{(1)}(\mathcal{P})=\max _{\gamma \in \mathcal{V}(\mathcal{P})}|\gamma|$ is the maximum order of $\mathcal{P}$;

$q(\mathcal{P})=\left(\frac{\mu(\mathcal{P})}{\mu_{1}(\mathcal{P})}, \ldots, \frac{\mu(\mathcal{P})}{\mu_{n}(\mathcal{P})}\right)$

$\forall \xi \in \mathbf{R}^{n}|\xi|_{\mathcal{P}}=\left(\sum_{s \in \mathcal{V}(\mathcal{P})} \xi^{2 s}\right)^{1 /(2 \mu)}$ is the weight associated to the polyhedron $\mathcal{P}$.

We observe that the following estimates are satisfied:

$$
\begin{aligned}
& \frac{|s|}{\mu} \leq k(s, \mathcal{P}) \leq|s| \quad \forall s \in \mathbf{R}_{+}^{n} ; \\
& (1+|\xi|)^{\mu^{(0)} / \mu} \leq|\xi|_{\mathcal{P}} \leq(1+|\xi|)^{\mu^{(1)} / \mu} \leq 1+|\xi| .
\end{aligned}
$$

Considering a polynomial with complex coefficients, we can regard it as the symbol of a differential operator, and associate a polyhedron to it, as in the following.

Definition 3. Let $P(D)=\sum_{|\alpha|<m} c_{\alpha} D^{\alpha}, c_{\alpha} \in \mathbf{C}$ be a differential operator with complex coefficients in $\mathbf{R}^{n}$ and $P(\xi)=\sum_{|\alpha| \leq m} c_{\alpha} \xi^{\alpha}, \xi \in \mathbf{R}^{n}$ its characteristic polynomial. The Newton polyhedron or characteristic polyhedron associated to $P(D)$ is the convex hull of the set

$$
\{0\} \cup\left\{\alpha \in \mathbf{Z}_{+}^{n}: c_{\alpha} \neq 0\right\} .
$$

There follow some examples of Newton polyhedra related to differential operators.

1. If $P(D)$ is an elliptic operator of order $m$, then its Newton polyhedron is complete and is the polyhedron of vertices $\left\{0, m e_{j}, j=1, \ldots, n\right\}$ and so $\mathcal{P}=\left\{\xi \in \mathbf{R}^{n}\right.$ : $\left.\xi \geq 0, \sum_{i=1}^{n} \xi_{i} \leq m\right\}$.

The set $\mathcal{N}_{1}(\mathcal{P})$ is reduced to one point $\nu=m^{-1} \sum_{j=1}^{m} e_{j}=\left(m^{-1}, \ldots, m^{-1}\right)$.

Moreover, $m_{j}(\mathcal{P})=\mu_{j}(\mathcal{P})=\mu^{(0)}(\mathcal{P})=\mu^{(1)}(\mathcal{P})=\mu(\mathcal{P})=m, j=1, \ldots, n$; 
$q(\mathcal{P})=(1,1, \ldots, 1) ; k(s, \mathcal{P})=m^{-1}|s|=m^{-1} \sum_{j=1}^{n} s_{j}, s \in \mathbf{R}_{+}^{n}$.

2. If $P(\xi)$ is a quasi-elliptic polynomial of order $m$ (see for example Hörmander [10], Rodino [14], Zanghirati [16]), its characteristic polyhedron $\mathcal{P}$ is complete and has vertices $\left\{0, m_{j} e_{j}, j=1, \ldots, n\right\}$, where $m_{j}$ are fixed integers.

The set $\mathcal{N}_{1}(\mathcal{P})$ is again reduced to one point: $\nu=\sum_{j=1}^{n} m_{j}^{-1} e_{j}$.

Moreover, $\mathcal{P}=\left\{\xi \in \mathbf{R}^{n}: \xi \geq 0, \sum_{j=1}^{n} m_{j}^{-1} \xi_{j} \leq 1\right\} ;$

$\mu_{j}(\mathcal{P})=m_{j}, \quad j=1, \ldots, n$

$\mu^{(0)}(\mathcal{P})=\min _{j=1, \ldots, n} m_{j}$;

$\mu(\mathcal{P})=\mu^{(1)}(\mathcal{P})=\max _{j=1, \ldots, n} m_{j}=m ;$

$q(\mathcal{P})=\left(\frac{m}{m_{1}}, \ldots, \frac{m}{m_{n}}\right) ; k(s, \mathcal{P})=\mu(\mathcal{P})^{-1} q \cdot s, \quad s \in \mathbf{R}_{+}^{n}$.

In this case the unique face of $\mathcal{P}$ is defined by the equation

$$
\frac{1}{m_{1}} x_{1}+\ldots+\frac{1}{m_{n}} x_{n}=1 \text {. }
$$

We now introduce a class of generalized Gevrey functions with weight given by a complete polyhedron, as in Corli [7], Zanghirati [17].

They can be regarded as a particular case of inhomogeneous Gevrey classes with weight $\lambda$, in the sense of the definition of Liess-Rodino [14], by choosing $\lambda(\xi)=|\xi|_{\mathcal{P}}$, cf. Theorem 3 below.

Following Corli [7] we give first the following definition in terms of the derivatives of $u$.

Definition 4 . Let $\mathcal{P}$ be a complete polyhedron in $\mathbf{R}^{n}$. Let $\Omega$ be an open set in $\mathbf{R}^{n}$ and $s \in \mathbf{R}, s>1$. We denote by $G^{s \mathcal{P}}(\Omega)$ the set of all $u \in C^{\infty}(\Omega)$ such that

for all $K \subset \subset \Omega$ there exists $C>0$ such that

$$
\forall \alpha \in \mathbf{Z}_{+}^{n}\left|D^{\alpha} u(x)\right| \leq C^{|\alpha|+1}(\mu k(\alpha, \mathcal{P}))^{s \mu k(\alpha, \mathcal{P})} .
$$

We also define

$$
G_{0}^{s \mathcal{P}}(\Omega)=G^{s \mathcal{P}}(\Omega) \cap C_{0}^{\infty}(\Omega) .
$$

The space $G^{s \mathcal{P}}(\Omega)$ can be endowed with a natural topology. Namely, we denote by $C^{\infty}(\mathcal{P}, s, K, C)$ the space of functions $u \in C^{\infty}(\Omega)$ such that

$$
\operatorname{supp} u \subset K, \quad\|u\|_{K, C}=\sup _{\alpha \in \mathbf{Z}_{+}^{n}} \sup _{x \in K} C^{-|\alpha|}(\mu k(\alpha, \mathcal{P}))^{-s \mu k(\alpha, \mathcal{P})}\left|D^{\alpha} u(x)\right|<\infty .
$$

With such a norm $C^{\infty}(\mathcal{P}, s, K, C)$ becomes a Banach space. Then

$$
G^{s \mathcal{P}}(\Omega)=\bigcap_{K \subset \subset \Omega} \bigcup_{C>0} C^{\infty}(\mathcal{P}, s, K, C)
$$

endowed with the topology of projective limit of inductive limit.

REMARK 1. If $\mathcal{P}$ is the Newton polyhedron of an elliptic operator, then $G^{s \mathcal{P}}(\Omega)$ coincides with $G^{s}(\Omega)$, the set of the standard $s$-Gevrey functions in $\Omega$.

REMARK 2. If $\mathcal{P}$ is the Newton polyhedron of a quasi-elliptic operator, then

$$
G^{s \mathcal{P}}(\Omega)=G^{s q}(\Omega), \quad \text { where } q=\left(\frac{m}{m_{1}}, \ldots, \frac{m}{m_{n}}\right),
$$


is the set of the anisotropic Gevrey functions, for definition see Hörmander [10], Rodino [14], Zanghirati [16].

REMARK 3. We have the following inclusion:

$$
\forall s>1 \forall \mathcal{P} \quad G^{s} \subset G^{s \mathcal{P}},
$$

as follows immediately from Definition 4 as $|\alpha| \leq \mu k(\alpha, \mathcal{P})$ for every $\mathcal{P}$.

More precisely, we have the chain of inclusions

$$
\forall s>1 \forall \mathcal{P} \quad G^{s \mu / \mu^{(1)}} \subset G^{s \mathcal{P}} \subset G^{s \mu / \mu^{(0)}},
$$

where the minimum order $\mu^{(0)}$ and the maximum order $\mu^{(1)}$ are defined as before. The inclusion is strict, except the elliptic case.

This implies in particular that for any $s>1$ fixed and any $r>1$ there is a complete polyhedron $\mathcal{P}$ such that $G^{r} \subset G^{s \mathcal{P}}$, in fact $\frac{\mu}{\mu^{(1)}}$ may be as large as we want for a suitable $\mathcal{P}$.

We give now equivalent definitions of generalized Gevrey classes. At the end, we obtain an estimate of the Fourier transform of a generalized Gevrey function with an exponential of the weight function. With respect to a similar estimate in the standard Gevrey classes, the proof is more complicated as we have to deal with the weight function associated to the polyhedron.

LEMMA 1. Let $s>1$, for every $K \subset \subset \Omega$ there is a function $\chi \in C_{0}^{\infty}\left(\mathbf{R}^{n}\right)$ such that

$$
\begin{aligned}
& \chi(x)=1, \quad x \in K, \\
& \left|D^{\alpha} \chi\right| \leq C\left(C N^{s \mu}\right)^{\alpha \cdot \nu}, \text { if } \alpha \cdot \nu \leq N, N=1,2, \ldots, \nu \in \mathcal{N}_{1}(\mathcal{P}) .
\end{aligned}
$$

Lemma 2. With the previous notation, if $u \in G^{s \mathcal{P}}\left(\mathbf{R}^{n}\right)$, then taking $\chi$ as in Lemma 1 , we obtain the estimate

$$
|\widehat{\chi u}(\xi)| \leq C\left(\frac{C N^{s}}{|\xi|_{\mathcal{P}}+N^{s}}\right)^{\mu N}, \quad N=1,2, \ldots
$$

By using the preceding two lemmas, we obtain the following results. For details and the proof see Calvo [3].

TheOREM 3. Let $\Omega$ be an open set in $\mathbf{R}^{n}, x_{0} \in \Omega, u \in \mathcal{D}^{\prime}(\Omega)$. Then $u$ is of the class $G^{s \mathcal{P}}$ in a neighborhood of $x_{0}$ if and only if there is a neighborhood $U$ of $x_{0}$ and a distribution $v \in \mathcal{E}^{\prime}(\Omega)$, or $v \in \mathcal{S}^{\prime}\left(\mathbf{R}^{n}\right)$, such that

1. $v=u$ in $U$,

2. $\hat{v}$ satisfies

$$
|\hat{v}(\xi)| \leq C\left(\frac{C N^{s}}{|\xi|_{\mathcal{P}}}\right)^{\mu N}=C\left(\frac{C^{\prime} N}{|\xi|_{\mathcal{P}}^{1 / s}}\right)^{s \mu N}, \quad N=1,2, \ldots
$$

THEOREM 4. We have the following characterization of $G^{s \mathcal{P}}$ by means of the Fourier transform:

1. Let $u \in G_{0}^{s \mathcal{P}}\left(\mathbf{R}^{n}\right)$. Then there exist two constants $C>0, \epsilon>0$ such that

$$
|\hat{u}(\xi)| \leq C \exp \left(-\epsilon|\xi|_{\mathcal{P}}^{1 / s}\right)
$$

2. If the Fourier transform of $u \in \mathcal{E}^{\prime}\left(\mathbf{R}^{n}\right)$, or $u \in \mathcal{S}^{\prime}\left(\mathbf{R}^{n}\right)$ satisfies (8), then $u \in$ $G^{s \mathcal{P}}\left(\mathbf{R}^{n}\right)$. 
2. Multi-quasi-hyperbolic operators. For any complete polyhedron $\mathcal{P}$ we define the corresponding class of multi-quasi-hyperbolic operators according to Definition 1. For short, we denote multi-quasi-hyperbolic operators of order $s$ with respect to $\mathcal{P}$ by $(s, \mathcal{P})$-hyperbolic.

There follow some properties of $(s, \mathcal{P})$-hyperbolic operators and some necessary and sufficient conditions of $(s, \mathcal{P})$-hyperbolicity. For the proofs of the next Propositions 5, 6, 7 we refer to $[3]$.

Proposition 5. If $P(D)$ is $(s, \mathcal{P})$-hyperbolic, $1<s<\infty$, then there is a constant $C>0$ such that for any $(\lambda, \xi) \in \mathbf{C} \times \mathbf{R}^{n}$ with $P(\lambda, \xi)=0$, we have

$$
|\Im \lambda| \leq C|\xi|_{\mathcal{P}}^{1 / s}
$$

Proposition 6. If $P(D)$ is $(s, \mathcal{P})$-hyperbolic, $1<s<\infty$, then the principal part $P_{m}(D)$ of $P(D)$ is hyperbolic, i.e. the homogeneous polynomial $P_{m}(\lambda, \xi)$ satisfies

$$
P_{m}(\lambda, \xi)=0, \quad(\lambda, \xi) \in \mathbf{C} \times \mathbf{R}^{n} \Rightarrow \Im \lambda=0 .
$$

Proposition 7. For a differential operator $P_{m}(D)$ associated to a homogeneous polynomial $P_{m}(\lambda, \xi)$, the notions of hyperbolicity and $(s, \mathcal{P})$-hyperbolicity coincide, for every $s$, $1<s<\infty$.

Proposition 8. Let $P(D)$ be a differential operator of the form

$$
P(D)=P_{m}(D)+\sum_{|\nu|+j \leq m-1} a_{\nu j} D_{x}^{\nu} D_{t}^{j}
$$

with homogeneous principal part

$$
P_{m}(D)=D_{t}^{m}+\sum_{|\nu|+j=m} b_{\nu j} D_{x}^{\nu} D_{t}^{j},
$$

hyperbolic and such that $P_{m}(\lambda, \xi)=0$ has roots of multiplicity less than or equal to $M$. Let the lower order terms satisfy

$$
\left|a_{\nu j} \xi^{\nu}\right| \leq C|\xi|_{\mathcal{P}}^{k}(1+|\xi|)^{m-M-j} \quad \text { for } \quad|\nu|+j \leq m-1, k<M .
$$

Then $P(D)$ is $\left(\frac{M}{k}, \mathcal{P}\right)$ hyperbolic.

To prove Proposition 8 we use the following lemma, for the proof see for example Mizohata [13].

Lemma 9. Let $P=P\left(D_{t}, D_{x}\right)$ be an hyperbolic operator in $\mathbf{R} \times \mathbf{R}^{n}$ with principal symbol $P_{m}(\lambda, \xi)$ whose roots have maximal multiplicity equal to $M$. Then there is a constant $C>0$ such that

$$
\left|P_{m}(\lambda, \xi)\right| \geq C(|\lambda|+|\xi|)^{m-M}|\Im \lambda|^{M} .
$$

Proof of Proposition 8. The roots $\lambda_{j}(\xi)(j=1, \ldots, m)$ of $P(\lambda, \xi)=0$ must satisfy for $\epsilon>0$ the inequality

$$
|\lambda| \leq \epsilon^{-1}|\xi|
$$

and for $\lambda_{j}(\xi)$ we can write

$$
P_{m}(\lambda, \xi)=-\left(P(\lambda, \xi)-P_{m}(\lambda, \xi)\right)=-\sum_{|\nu|+j \leq m-1} a_{\nu j} \xi^{\nu} \lambda^{j}
$$


So, by the hypothesis (13) and the estimate (15) we obtain for $P_{m}(\lambda, \xi)$

$$
\begin{aligned}
\left|P_{m}(\lambda, \xi)\right| \leq & \sum_{|\nu|+j \leq m-1}\left|a_{\nu j} \xi^{\nu} \lambda^{j}\right| \\
& \leq C \sum_{|\nu|+j \leq m-1}|\xi|_{\mathcal{P}}^{k}(1+|\xi|)^{m-M-j}|\lambda|^{j} \leq C^{\prime}|\xi|_{\mathcal{P}}^{k}(1+|\xi|)^{m-M}
\end{aligned}
$$

By Lemma 9 we have also for large $\lambda$

$$
\left|P_{m}(\lambda, \xi)\right| \geq C(|\lambda|+|\xi|)^{m-M}|\Im \lambda|^{M} \geq C(1+|\xi|)^{m-M}|\Im \lambda|^{M} .
$$

Both together give

$$
|\Im \lambda|^{M}(1+|\xi|)^{m-M} \leq C^{\prime \prime}|\xi|_{\mathcal{P}}^{k}(1+|\xi|)^{m-M}
$$

and this implies

$$
|\Im \lambda| \leq C^{\prime \prime}|\xi|_{\mathcal{P}}^{M / k}
$$

i.e. $P=P\left(D_{x}, D_{t}\right)$ is $\left(\frac{M}{k}, \mathcal{P}\right)$-hyperbolic as we wanted to prove.

Proposition 10. Any differential operator $P(D)=D_{t}^{m}+\sum_{|\nu|+j \leq m-1} a_{\nu j} D_{x}^{\nu} D_{t}^{j}$ satisfying the condition

$$
\left|a_{\nu j} \xi^{\nu}\right| \leq C|\xi|_{\mathcal{P}}^{k-j} \quad \text { for }|\nu|+j \leq m-1, k<m
$$

is $\left(\frac{m}{k}, \mathcal{P}\right)$ hyperbolic.

We note that the principal part is only $D_{t}^{m}$ and is obviously hyperbolic. Proposition 10 states that in this particular case we may replace (13) by the weaker assumption (19).

PROOF. In this case the roots of $P(\lambda, \xi)$ satisfy for $\epsilon>0$ the inequality

$$
|\lambda| \leq \epsilon^{-1}|\xi|_{\mathcal{P}}
$$

For $(\lambda, \xi)$ satisfying $P(\lambda, \xi)=0$ we write

$$
\lambda^{m}=-\left(P(\lambda, \xi)-\lambda^{m}\right)=-\sum_{|\nu|+j \leq m-1} a_{\nu j} \xi^{\nu} \lambda^{j} .
$$

In view of the estimate (20) for $\lambda$ and (19) for $a_{\nu j} \xi^{\nu}$ we conclude

$$
|\lambda|^{m} \leq C^{\prime} \sum_{j=0}^{m-1}|\xi|_{\mathcal{P}}^{k-j}|\lambda|^{j} \leq C^{\prime \prime}|\xi|_{\mathcal{P}}^{k}
$$

Hence,

$$
|\Im \lambda|^{m} \leq C^{\prime \prime}|\xi|_{\mathcal{P}}^{k}, \quad|\Im \lambda| \leq C^{\prime \prime \prime}|\xi|_{\mathcal{P}}^{k / m}
$$

i.e. $P(D)$ is $\left(\frac{m}{k}, \mathcal{P}\right)$ hyperbolic.

REMARK 4. A more general version of Proposition 10 is easily obtained by assuming as in Proposition 8 that $P(D)$ has a hyperbolic homogeneous principal part $P_{m}(D)=$ $\sum_{|\nu|+j=m} b_{\nu j} D_{x}^{\nu} D_{t}^{j}$ with

$$
\left|b_{\nu j} \xi^{\nu}\right| \leq C|\xi|_{\mathcal{P}}^{m-j}, \quad j=0,1, \ldots, m-1
$$

and still keeping condition (19) for the lower order terms.

Observe however that (21) implies $b_{\nu j}=0$ for all $j$, except the quasi-homogeneous case. 
There follow some examples of multi-quasi-hyperbolic operators. The result is similar to Proposition 8 for systems as in Calvo [3]. The analogue of Proposition 10 to systems will be presented in a forthcoming paper.

REMARK 5. Proposition 12 in the next section will give for these examples a result of well-posedness for data in generalized Gevrey classes, which are strictly larger than the standard Gevrey classes, as obtained in [4], [10], [14].

1. If $P(D)$ is a differential operator in $\mathbf{R}^{n}$ with symbol $P(\xi)$ and complete Newton polyhedron $\mathcal{P}$ of formal order $\mu$, then the differential operator in $\mathbf{R}^{n+1}$

$$
Q(D)=D_{t}^{m}+P\left(D_{x}\right)
$$

with $m>\mu$, is multi-quasi-hyperbolic of order $\frac{m}{\mu}$ with respect to $\mathcal{P}$.

In fact, the roots of the symbol of $Q(D)$ satisfy

$$
|\Im \lambda| \leq C|\xi|_{\mathcal{P}}^{\mu / m}
$$

Let us observe that, arguing in the standard Gevrey classes $G^{s}$, and applying the classical result of $s$-hyperbolicity mentioned in the introduction (see [2], [4], [5], [10], [11], [14]), we would obtain for the previous operator well-posedness in $G^{m / \mu^{(1)}}$, where $\mu^{(1)}$ is the maximal order of $\mathcal{P}$, i.e. the classical order of $P\left(D_{x}\right)$. Since according to Remark 3 we have $G^{m / \mu^{(1)}} \subset G^{m / \mu, \mathcal{P}}$, our result is stronger, providing a larger class of admissible data. Observe however that the classical $G^{s}$ result keeps valid for $m>\mu^{(1)}$, whereas we may argue only under the stronger condition $m>\mu$.

2. A particular case of Proposition 8 is: if $\mathcal{P}$ is the polyhedron in $\mathbf{R}^{2}$ with vertices $(0,0),(0,2),(1,0)$, then $\mu=2$ and the operator

$$
P\left(D_{x_{1}}, D_{x_{2}}, D_{t}\right)=P_{3}\left(D_{x_{1}}, D_{x_{2}}, D_{t}\right)+C_{1} D_{x_{2}}^{2}+C_{2} D_{x_{1}}+C_{3} D_{x_{2}}+C_{4} D_{t}+C_{5}
$$

where $P_{3}\left(D_{x_{1}}, D_{x_{2}}, D_{t}\right)$ is a hyperbolic homogeneous operator of order 3 and $C_{1}, \ldots, C_{5}$ belong to $\mathbf{C}$, is multi-quasi-hyperbolic of order $\frac{3}{2}$ with respect to $\mathcal{P}$.

3. If $\mathcal{P}$ is the same polyhedron as in Example 2, then the operator of order 3

$$
\begin{aligned}
& P\left(D_{x_{1}}, D_{x_{2}}, D_{t}\right)=P_{3}\left(D_{x_{1}}, D_{x_{2}}, D_{t}\right) \\
& \quad+C_{1} D_{x_{1}} D_{x_{2}}+C_{2} D_{x_{2}}^{2}+C_{3} D_{x_{2}} D_{t}+C_{4} D_{x_{1}}+C_{5} D_{x_{2}}+C_{6} D_{t}+C_{7},
\end{aligned}
$$

where $C_{1}, \ldots C_{7} \in \mathbf{C}$ and the principal part $P_{3}\left(D_{x_{1}}, D_{x_{2}}, D_{t}\right)$ is hyperbolic and has roots of maximal multiplicity equal to 2 , is $(2, \mathcal{P})$-hyperbolic. In fact, its terms satisfy (13).

4. Another particular case of Proposition 8 is: if $\mathcal{P}$ is the polyhedron in $\mathbf{R}^{2}$ with vertices $(0,0),(0,3),(1,2),(2,0)$, then the formal order is $\mu=4$ and the operator of order 4

$$
\begin{array}{r}
P\left(D_{x_{1}}, D_{x_{2}}, D_{t}\right)=P_{4}\left(D_{x_{1}}, D_{x_{2}}, D_{t}\right)+C_{1} D_{x_{2}}^{2}+C_{2} D_{x_{1}} D_{x_{2}}+C_{3} D_{x_{2}} D_{t} \\
+C_{4} D_{x_{1}}+C_{5} D_{x_{2}}+C_{6} D_{t}+C_{7}
\end{array}
$$

where $P_{4}\left(D_{x_{1}}, D_{x_{2}}, D_{t}\right)$ is a hyperbolic homogeneous operator of order 4 and $C_{1}, \ldots, C_{7}$ belong to $\mathbf{C}$, is multi-quasi-hyperbolic of order $\frac{4}{3}$ with respect to $\mathcal{P}$.

5 . Let $\mathcal{P}$ be the same polyhedron as in Example 4. Then any operator of order 5 of the form

$$
P\left(D_{x_{1}}, D_{x_{2}}, D_{t}\right)=P_{5}\left(D_{x_{1}}, D_{x_{2}}, D_{t}\right)
$$




$$
+C_{1} D_{x_{2}}^{3}+C_{2} D_{x_{2}}^{2} D_{t}+C_{3} D_{x_{1}} D_{x_{2}}^{2}+P_{2}\left(D_{x_{1}}, D_{x_{2}}, D_{t}\right)
$$

with hyperbolic principal part $P_{5}\left(D_{x_{1}}, D_{x_{2}}, D_{t}\right)$, having roots of multiplicity less than or equal to 4 , where $P_{2}\left(D_{x_{1}}, D_{x_{2}}, D_{t}\right)$ denotes a generic operator with constant coefficients of order 2 and $C_{1}, \ldots, C_{3} \in \mathbf{C}$, is $\left(\frac{4}{3}, \mathcal{P}\right)$-hyperbolic as its terms satisfy condition (13).

3. Well-posedness in generalized Gevrey classes. We now present results of well-posedness of the Cauchy problem in generalized Gevrey classes for multi-quasihyperbolic operators. First we give a result of regularity with respect to the space variables. Then we extend the regularity to the time variable. To do so, we need to extend the polyhedron to one more dimension. In this connection we shall present two theorems. The first one allows a better result in a particular situation. Then the general case is presented. For brevity, we omit the proofs, that are based on the properties of complete polyhedra, on the associated weight functions, on the properties of the Fourier transform and on the generalized Gevrey functions. For details see Calvo [3].

TheOREM 11. Let $P(D)$ be a differential operator in $\mathbf{R}_{t} \times \mathbf{R}_{x}^{n}$ and let us consider the corresponding Cauchy problem

$$
\begin{aligned}
& P(D) u=D_{t}^{m} u+\sum_{|\nu|+j \leq m} a_{\nu+j} D_{x}^{\nu} D_{t}^{j} u=0, \\
& D_{t}^{k} u(0, x)=f_{k}(x), \quad x \in \mathbf{R}^{n}, k=0,1, \ldots, m-1,
\end{aligned}
$$

and let $P$ be multi-quasi-hyperbolic of order $s$ with respect to a complete polyhedron $\mathcal{P}$ in $\mathbf{R}^{n}$, with $1<s<\infty$. Let $1<r<s$ and assume $f_{k} \in G_{0}^{r \mathcal{P}}\left(\mathbf{R}_{x}^{n}\right)$ for $k=0,1, \ldots, m-1$. Then there exists a unique solution $u(t, \cdot) \in G^{r \mathcal{P}}\left(\mathbf{R}_{x}^{n}\right)$, for $t \in \mathbf{R}$, satisfying (22).

Now we will discuss the regularity with respect to the time variable. For this reason we extend the polyhedron to $(n+1)$ variables, that is possible by means of the following proposition:

Proposition 12. Given a complete polyhedron $\mathcal{P}$ in $\mathbf{R}^{n}$, we define $\mathcal{P}^{\prime}$ as the convex hull in $\mathbf{R}^{n+1}$ of the vertices of $\mathcal{P}$, regarded as points in $\mathbf{R}^{n+1}$ with zero as first component, plus the vector $\left(\mu_{0}, 0, \ldots, 0\right)$ with $\mu_{0} \in \mathbf{Q}_{+}, 0<\mu_{0} \leq \mu$. Then $\mathcal{P}^{\prime}$ is a complete polyhedron in $\mathbf{R}^{n+1}$ with the same formal order $\mu$ of $\mathcal{P}$. We call $\mathcal{P}^{\prime}$ an extension of $\mathcal{P}$ in $\mathbf{R}^{n+1}$.

If the further vertex has coordinates $(\mu, 0, \ldots, 0)$ with $\mu$ denoting the formal order of $\mathcal{P}$, we say that $\mathcal{P}^{\prime}$ is the maximal extension of $\mathcal{P}$ in $\mathbf{R}^{n+1}$.

Now we present two results for regularity with respect to the time variable.

THEOREM 13. Let us suppose the assumptions of Theorem 11. If the terms $a_{\nu j} \xi^{\nu}$ satisfy the condition

$$
\left|a_{\nu j} \xi^{\nu}\right| \leq C|\xi|_{\mathcal{P}}^{m-j}, \quad j=0,1, \ldots, m-1,
$$

then the solution $u$ of the Cauchy problem (22) belongs to the class $G^{r \mathcal{P}^{\prime}}\left(\mathbf{R}^{n+1}\right)$, where $\mathcal{P}^{\prime}$ denotes the maximal extension of $\mathcal{P}$ to $\mathbf{R}^{n+1}$.

In the general case, we have the following result, that allows to consider a smaller Gevrey class, as we ask for a stronger condition on the polyhedron $\mathcal{P}^{\prime}$.

THEOREM 14. Under the assumptions of Theorem 11 the solution $u$ to the Cauchy problem (22) belongs to $G^{r \mathcal{P}^{\prime}}\left(\mathbf{R}^{n+1}\right)$, where $\mathcal{P}^{\prime}$ is the extension of $\mathcal{P}$ to $\mathbf{R}^{n+1}$ which is 
obtained by adding to $\mathcal{P}$ the vertex

$$
\begin{aligned}
& s_{0}=\left(\mu_{0}, 0, \ldots, 0\right), \mu_{0}=\mu^{(0)}, \\
& \mu^{(0)}=\mu^{(0)}(\mathcal{P})=\min \left\{m_{j}: m_{j} e_{j} \in \mathcal{V}(\mathcal{P}), j=1, \ldots, n\right\}=\min _{\gamma \in \mathcal{V}(\mathcal{P}) \backslash\{0\}}|\gamma| .
\end{aligned}
$$

\section{References}

[1] P. Boggiatto, E. Buzano, L. Rodino, Global Hypoellipticity and Spectral Theory, Akademie Verlag, Berlin, 1996.

[2] M. D. Bronstein, The Cauchy problem for hyperbolic operators with characteristics of variable multiplicity, Trudy Moskov. Mat. Obshch. 41 (1980), 83-99 (in Russian); English transl.: Trans. Moscow Math. Soc. 41 (1980), 87-103.

[3] D. Calvo, Generalized Gevrey classes and multi-quasi-hyperbolic operators, Rend. Sem. Mat. Univ. Politec. Torino (to appear).

[4] L. Cattabriga, Alcuni problemi per equazioni differenziali lineari con coefficienti costanti, Quaderni Unione Matematica Italiana 24, Pitagora, Bologna, 1983.

[5] L. Cattabriga, Moltiplicatori di Fourier e teoremi di immersione per certi spazi funzionali, Ann. Scuola Norm. Sup. Pisa (3) 24 (1970), 111-158.

[6] L. Cattabriga, D. Mari, Parametrix of infinite order on Gevrey spaces to the Cauchy problem for hyperbolic operators with one constant multiple characteristic, Ricerche Mat. 36 (1987), Suppl., 127-147.

[7] A. CoRLI, Un teorema di rappresentazione per certe classi generalizzate di Gevrey, Boll. Un. Mat. Ital. C(6) 4 (1985), 245-257.

[8] J. Friberg, Multi-quasielliptic polynomials, Ann. Scuola Norm. Sup. Pisa (3) 21 (1967), 239-260.

[9] G. H. Hakobyan, V. N. Margaryan, On Gevrey Type Solutions of Hypoelliptic Equations, Izv. Nats. Akad. Nauk Armenii Mat. 31 (1996), No. 2, 40-56 (in Russian); English transl.: J. Contemp. Math. Anal. 31 (1996), No. 2, 33-47.

[10] L. Hörmander, The Analysis of Linear Partial Differential Operators, I, II, III, IV, Springer, Berlin, 1983-1985.

[11] K. KaJitani, Local solution of the Cauchy problem for nonlinear hyperbolic systems in Gevrey classes, Hokkaido Math. J. 12 (1983), 434-460.

[12] O. Liess, L. Rodino, Inhomogeneous Gevrey classes and related pseudo-differential operators, Boll. Un. Mat. Ital. C(6) 3 (1984), 233-323.

[13] S. Mizonata, The Theory of Partial Differential Equations, Cambridge University Press, New York, 1973.

[14] L. Rodino, Linear Partial Differential Operators in Gevrey Spaces, World Scientific, Singapore, 1993.

[15] S. Steinberg, Existence and uniqueness of solutions of hyperbolic equations which are not necessarily strictly hyperbolic, J. Differential Equations 17 (1975), 119-153.

[16] L. ZANGHIRATI, Iterati di una classe di operatori ipoellittici e classi generalizzate di Gevrey, Boll. Un. Mat. Ital. Suppl. 1980, no. 1, 177-195.

[17] L. Zanghirati, Iterati di operatori e regolarità Gevrey microlocale anisotropa, Rend. Sem. Mat. Univ. Padova 67 (1982), 85-104. 